\title{
OThI6.pdf
}

\section{An Optimal Model for LSP Bundle Provisioning in PCE-based WDM Networks}

\author{
J. Ahmed, C. Cavdar, P. Monti, L. Wosinska \\ The Royal Institute of Technology (KTH), Stockholm, Sweden \\ \{jawwad, cavdar, pmonti, wosinska\}@kth.se
}

\begin{abstract}
A time-efficient resource optimization model for dynamic concurrent provisioning of connection requests at PCE is proposed. It is shown that a significant performance improvement can be achieved without noticeable increase in connection setup-time.

(C) 2011 Optical Society of America

OCIS codes: (060.0060) Fiber optics and optical communications; (060.4250) Networks
\end{abstract}

\section{Introduction}

Recently networks utilizing Path Computation Element (PCE) [1] has turned out to be a viable alternative where a PCE serves as a central entity capable of solving complex, multi-constrained Label Switched Path (LSP) computation problems. The PCE concept was validated in an experimental network [2, 3], where the performance of the path computation procedure was evaluated and results demonstrated efficient resource utilization and a good network scalability of the PCE-based approach. However, this centralized approach may suffer some control and signaling overheads in highly dynamic network scenarios. One possible way to overcome these problems is to take advantage of some implementation features supported by the Path Computation Element communication Protocol (PCEP) [4], in particular the one that allows for bundling of LSP path computation requests [5].

In the PCE paradigm, communication between a node and a PCE is provided by the PCReq and PCRep messages specified for the PCEP protocol. In a single PCReq message several LSP requests can be bundled together by PCC (Path Computation Client) before being sent to the PCE. Similarly several path computation responses can be bundled in a single PCRep message in the opposite direction. This bundling feature not only reduces the control plane overhead, but also can be exploited to achieve significant improvements in terms of overall network performance, i.e., enabling the concurrent optimization of several LSP requests with remarkable benefits in terms of blocking probability and resource utilization [6]. All the performance results for the PCEP bundling obtained in the literature so far are obtained with routing solutions based on heuristic algorithms. An assessment against an optimal routing benchmark, on the other hand, is needed to demonstrate the full potentials of the bundling approach.

In this paper a novel and scalable ILP model suitable for the dynamic bundle provisioning problem is proposed. The network scenario under consideration is as following. A time-threshold is used to collect LSP requests at each ingress node to bound the maximum waiting time. After the timer expires PCC packs the LSP requests in a single PCReq message forming a bundle and sends it to the PCE using the PCEP protocol. In addition, at the PCE, a counter based threshold (BundleThrsh\#) is employed to ensure that a specific number of PCReq messages are collected before the path computation phase starts. After collecting the required number of PCReq messages, all the LSP requests are used to form a bulk and are then fed to an ILP solver. The resulting paths calculated via the ILP solver are bundled into PCRep messages where all the LSP requests originating at the same PCC are packed in the same PCRep message.

Simulation results demonstrate that significant performance gains can be achieved by using the proposed ILP model at the PCE for path computation. Values of BundleThrsh\# $>1$ are particularly useful to reduce blocking since they enable PCE to process LSP requests from a more diverse set of ingress nodes. Furthermore, it is shown that moderate sized LSP bundles can be processed efficiently without a noticeable increase in the LSP setup-time.

\section{LSP Bundle Provisioning Problem Formulation}

After the PCE collects a certain number of PCReq messages, each containing a set of LSP requests, it opens each bundle and it solves the concurrent optimization problem for the set of new arrivals which can be stated as follows:

Given:

$G(N, E)$ : physical topology consisting of set of nodes $N$ and set of links $E$. $W$ : maximum \# of wavelengths supported on each link. $W_{x y}^{w}$ : is 1 if wavelength $w$ on link $N(x, y)$ is available. $W_{x y}=\sum_{\forall w} W_{x y}^{w}$ : number of free wavelengths on link $(x, y)$. D: set of LSP requests to be provisioned by the PCE, where $\lambda_{c} \in D$ denotes an LSP request $c$ from source $s$ to destination $d$.

Find:

Binary variable $F_{x y, w}^{c}$ : is 1 if LSP request $c$ is routed through link $(x, y)$ on wavelength $w$. Binary variable $A_{c}:$ is 1

This work was supported by the Network of Excellence "Building the Future Optical Network in Europe" (BONE), funded by the European Commission through the 7th ICT-Framework Programme. 


\section{OThI6.pdf}

if LSP request $c$ is successfully provisioned and $A_{c}^{w}$ is 1 if LSP request $c$ uses wavelength $w$. M denotes the load of the maximally loaded link in the network. $L_{x y}$ denotes the wavelength load of link $(x, y)$.

The objective of the problem is shown below as a weighted sum with different weights denoted by $\alpha, \beta, \gamma$ where $\alpha>>\beta>>\gamma$ in order to give priority to the minimization of the first term. The first term aims at minimizing the LSP blocking rate, the second term corresponds to capacity efficiency and the last term ensures load balancing. Note that $c$ denotes a request in the LSP demand set i.e., $c \in D$ from a source $s$ to destination $d$ while $(x, y)$ denotes the nodes of a physical link, i.e., $(x, y) \in E$ and $w$ denotes a wavelength, with $w \in W$ in all the following formulations.

Minimize $\alpha \cdot\left(|D|-\sum_{\forall c} A_{c}\right)+\beta \cdot \sum_{\forall(x, y)} L_{x y}+\gamma \cdot M$

Subject to

$$
\begin{aligned}
& \sum_{\forall x} F_{x y, w}^{c}-\sum_{\forall x} F_{y x, w}^{c}=\left\{\begin{array}{cl}
0, & y \neq s, d \\
-A_{c}^{w}, & y=s \\
A_{c}^{w}, & y=d
\end{array} \quad, \forall w, \forall c, \forall y\right. \\
& \sum_{\forall w} F_{x y, w}^{c} \leq 1, \forall c,(x, y) \\
& \sum_{\forall w} A_{c}^{w} \leq A_{c}, \forall c \\
& \sum_{\forall c} F_{x y, w}^{c} \leq 1, \forall w,(x, y) \\
& A_{c} \leq \lambda_{c}, \forall c \\
& \sum_{\forall w} \sum_{\forall c} F_{x y, w}^{c}=L_{x y}, \forall(x, y) \\
& L_{x y} \leq W_{x y}, \forall(x, y) \\
& F_{x y, w}^{c} \leq W_{x y}^{w}, \forall c, \forall w, \forall(x, y) \\
& M \geq L_{x y}, \forall(x, y)
\end{aligned}
$$

Constraint (1) is the flow conservation and the wavelength continuity constraint for routing request $\lambda_{c}$ from node $s$ to $d$. $A_{c}$ keeps track of the successfully routed connections. Constraints (2) and (3) ensure that each LSP can only employ one wavelength on a link and constraint (4) ensures that each $w$ can be used by only one LSP. The successful provisioning variable is bound by whether an LSP request $c$ exists in constraint(5). Wavelength link load on link $(x, y)$ is defined in constraint (6) while each link load is bound by the link capacity by constraint (7). Constraint (8) serves for eliminating the decision variables when $w$ on link $(x, y)$ is not available. The load of the maximally loaded link is defined in constraint (9) which helps load balancing as a tertiary objective.

\section{Simulation setup and numerical results}

Simulations studies are performed using a Java based discrete event-driven simulator. The network of reference is the NSF network topology [7] with 14 nodes and 20 links. Each link in the network is bidirectional with one fiber and 16 wavelengths for each direction. It is assumed that the wavelength-continuity-constraint is enforced. Two scenarios are considered for the LSP requests processing: (i) Sequential, when the LSP requests in a PCReq message are processed at the PCE in a sequential manner (i.e., in the order of arrival) and (ii) Concurrent, when these requests are processed in a combinatorial manner (i.e., based on an ILP formulation of the combinatorial RWA problem). It is assumed that the LSP requests in each bundle are synchronized (from a concurrent optimization point of view) using the SVEC (Synchronization Vector) object in the PCReq messages. For the sequential case, routes for the LSP requests are computed using the WLCR (Weighted Least Congested Routing) algorithm [7] and a First-Fit approach is used for the wavelength assignment. For the ILP case values of $\alpha, \beta$ and $\gamma$ are set to 1000.0, 1.0 and 0.1 respectively. The arrival rate $(\lambda)$ for the LSP requests follows a Poisson distribution and their service time $(1 / \mu)$ is assumed to be exponentially distributed. RSVP-TE signaling protocol is assumed to reserve the resources for a computed path. For the signaling phase the switch configuration time ( $T s$ ) at each node is assumed to be $50 \mathrm{~ms}$, and node processing delay ( $T p$ ) is assumed to be $20 \mathrm{~ms}$. The performance metrics considered in this work are: blocking probability (BP), average link utilization, PCEP control overhead, LSP setup-time and RWA computational time (i.e. time required to solve the RWA problem at the PCE) per LSP. The estimation of the PCEP control overhead, comprising also the signaling part, includes TCP, IP and Ethernet overhead, assuming that the control-plane is implemented over Ethernet. The LSP setup-time includes: path computation, PCEP protocol communication, queuing time at the PCE, and the signaling time. For benchmarking purposes a Baseline approach is also presented where LSP requests are sent one by one (i.e., no PCEP bundling) to the PCE and provisioned in sequence as they are received. It is assumed that $1 / \mu=80 \mathrm{~s}$, while $\lambda=1 / 150$ arrivals per second, per node pair, resulting in a total-load in 


\section{OThI6.pdf}

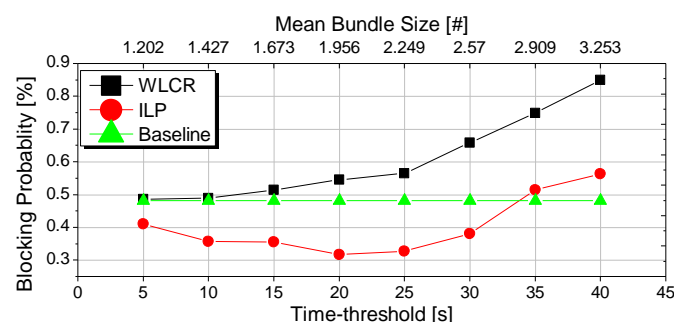

(a)

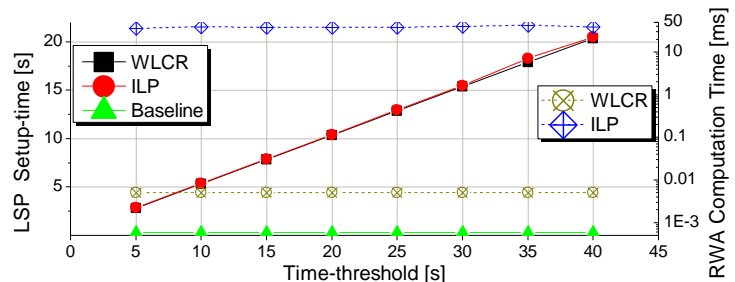

(c)
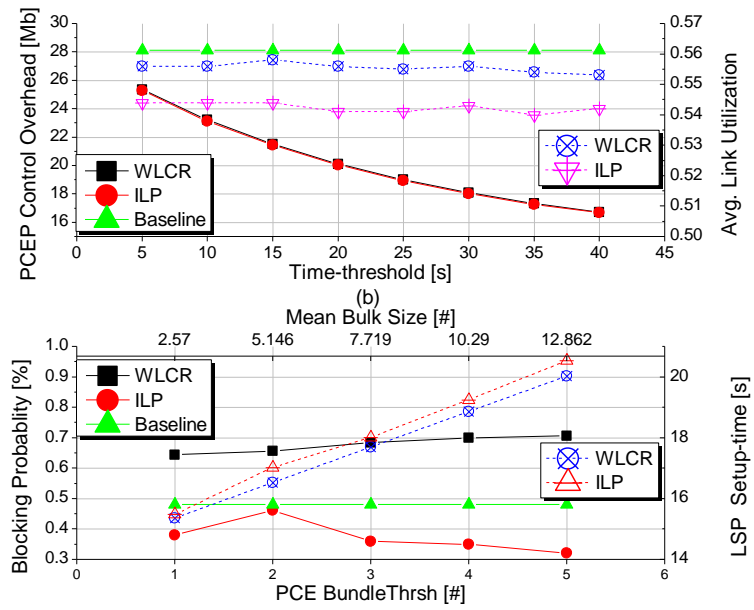

(d)

Fig.1. Blocking Probability vs. Time-threshold \& Mean Bundle Size (a), PCEP Control Overhead \& Avg. Link Utilization vs. Time-threshold (b), LSP Setup-time \& RWA Computation-Time vs. Time-threshold (c), Blocking Probability \& LSP Setup-time vs. BundleThrsh (d).

the network equal to 97 Erlang. Simulations were conducted in a Red Hat Enterprise Linux workstation fitted with $12 \mathrm{~GB}$ of memory and with a dual Intel Xeon CPUs (4 cores per CPU) clocked at $2.0 \mathrm{GHz}$.

Fig. 1(a) shows that a significant reduction of BP can be achieved using the proposed ILP model already with a time-threshold of $5 \mathrm{~s}$. The BP reduction continues to increase with increasing values of the time-threshold (e.g., more than $40 \%$ for a time-threshold of 30s). This trend stops at $30 \mathrm{~s}$ when BP starts to rapidly increase. This is due to the larger bundle sizes that increase the instantaneous network load, i.e., the traffic from a specific source node tends to become burstier. However, for time-thresholds values lower than $30 \mathrm{~s}$ this effect is weaker when compared to the gain provided by the concurrent optimization. For $W L C R$ the trend is similar but with a steeper curve. Fig. 1(a) also shows the mean bundle size which is the average number of LSP requests processed by the PCE in each bulk provisioning phase. Fig. 1(b) shows a significant control overhead reduction (28\%) with a time-threshold value of $30 \mathrm{~s}$. The figure also shows that the ILP solution makes a more efficient use of the wavelength resources. The LSP setup-time (Fig. 1(c)) increases linearly with the time-threshold value for both the ILP and the WLCR case. The figure also shows that the gap between the two approaches tends to be negligible. This proves that proposed ILP solution is an attractive and viable choice to be deployed in real PCE-based WDM network scenarios. Note that although the actual RWA computation time (Fig.1(c)) is significantly higher for the ILP solution as compared to the $W L C R$, it is still in the order of a few tens of ms for each successful LSP request. Based on these results, a value of the time-threshold of $30 \mathrm{~s}$ is chosen to investigate the effect of the BundleThrsh\# at the PCE (Fig. 1(d)) in terms of BP and LSP setup-time. Note that BundleThrsh\# is set to 1 except the results in Fig. 1(d). In Fig. 1(d), BP tends to decrease with an increasing number of LSP requests in one bulk for the ILP solution because higher values of BundleThrsh\# allows to process requests from a more diverse set of ingress nodes. The opposite is true for WLCR because of the limitation of sequential processing of LSP requests. However with bigger bulks the LSP setup-time increases, while the difference between the ILP solution and WLCR in terms of setup-time is more noticeable but still not significant $(<0.6 \mathrm{~s})$. So, a good value of the BundleThrsh\# is a trade-off between BP and LSP setup-time, and is dependent on the maximum tolerable LSP setup-time for a given scenario.

\section{Conclusion}

In this study an ILP based model suitable for dynamic bundle provisioning of LSP requests at the PCE was proposed. The benefits of the concurrent optimization were demonstrated in terms of BP and resource consumption in a PCE-based WDM network. The feasibility of the model in terms of network scalability was also confirmed by the numerical results (i.e., LSP setup-times and RWA computation time) when compared to a sequential solution.

\section{References}

[1] A. Farrel, et al., "A Path Computation Element (PCE) Based Architecture", IETF RFC 4655, Aug. 2006.

[2] F. Cugini, et al., in proc. of OFC/NFOEC 2007, Anaheim, CA, USA, March, 2007.

[3] P. Castoldi, et al., in proc. of ONDM 2007, Athens, Greece, May, 2007.

[4] J.-P. Vasseur and J. L. Le Roux, "Path Computation Element (PCE) Communication Protocol (PCEP)", Internet draft, Nov. 2008.

[5] J. Ahmed, et. al., in proc. of OFC/NFOEC 2009, San Diego, CA, USA, March 2009.

[6] J. Ahmed, et. al., in proc. of NOC/OC\&I 2009, Valladolid, Spain, June 2009.

[7] X.-W. Chu, et al., in proc. of IEEE INFOCOM 2003. San Francisco, CA, USA, April, 2003. 